\title{
Weak and strong convergence theorems of fixed points for nonexpansive mappings and strongly pseudocontractive mappings in a new class of probabilistic normed spaces
}

Yongchun $\mathrm{Xu}^{1 *}$, Jinyu Guan ${ }^{1}$ and Yongfu Su${ }^{2}$

*Correspondence:

hbxuyongchun@163.com 1 Department of Mathematics, Hebei North University, Zhangjiakou, 075000, China Full list of author information is available at the end of the article

\section{算 Springer}

\begin{abstract}
The purpose of this paper is to present a new class of probabilistic normed spaces and to study fixed point problems in this class of probabilistic normed spaces. This paper includes the following two contents: (1) The definition of a new class of probabilistic normed spaces, the so-called S-probabilistic normed spaces, is given. In order to study the fixed point problems, some relevant properties of S-probabilistic normed spaces are discussed and some basic useful results are obtained; (2) The notion of probabilistic weak convergence is firstly presented in this paper. Therefore the probabilistic weak and strong convergence theorems of fixed points for nonexpansive mappings, asymptotically nonexpansive mappings and strongly pseudocontractive mappings are also proved by using the new methods and techniques.
\end{abstract}

Keywords: probabilistic normed spaces; nonexpansive mappings; strongly pseudocontractive mappings; fixed point; weak convergence; iterative method

\section{Introduction and preliminaries}

Probabilistic metric spaces were introduced in 1942 by Menger [1]. In such spaces, the notion of distance between two points $x$ and $y$ is replaced by a distribution function $F_{x, y}(t)$. Thus one thinks of the distance between points as being probabilistic with $F_{x, y}(t)$ representing the probability that the distance between $x$ and $y$ is less than $t$. Sehgal, in his $\mathrm{PhD}$ thesis [2], extended the notion of a contraction mapping to the setting of Menger probabilistic metric spaces. For example, a mapping $T$ is a probabilistic contraction if $T$ is such that for some constant $0<k<1$, the probability that the distance between image points $T x$ and $T y$ is less than $k t$ is at least as large as the probability that the distance between $x$ and $y$ is less than $t$. Probabilistic normed spaces were introduced by Šerstnev in a series of papers [3-6]. Then a new definition was proposed by Alsina, Schweizer and Sklar [7]. This new definition revived the study of probabilistic normed spaces. The properties of these spaces were studied by several authors; here we shall mention [8-11], but see also the survey papers $[12,13]$.

Next we shall recall some well-known concepts and definitions which will be used later in this paper. For more details, we refer the reader to [14].

(c) $2015 \mathrm{Xu}$ et al. This article is distributed under the terms of the Creative Commons Attribution 4.0 International License (http://creativecommons.org/licenses/by/4.0/), which permits unrestricted use, distribution, and reproduction in any medium, provided you give appropriate credit to the original author(s) and the source, provide a link to the Creative Commons license, and indicate if changes were made. 
Definition 1.1 A triangular norm (shorter $\Delta$-norm) is a binary operation $\Delta$ on $[0,1]$ which satisfies the following conditions:

(a) $\Delta$ is associative and commutative;

(b) $\triangle$ is continuous;

(c) $\triangle(a, 1)=a$ for all $a \in[0,1]$;

(d) $\triangle(a, b) \leq \triangle(c, d)$ whenever $a \leq c$ and $b \leq d$ for each $a, b, c, d \in[0,1]$.

The following are the three basic $\Delta$-norms:

$$
\begin{aligned}
& \triangle_{1}(a, b)=\max (a+b-1,0) ; \\
& \triangle_{2}(a, b)=a \cdot b ; \\
& \triangle_{3}(a, b)=\min (a, b) .
\end{aligned}
$$

It is easy to check that the above three $\triangle$-norms have the following relations:

$$
\triangle_{1}(a, b) \leq \triangle_{2}(a, b) \leq \triangle_{3}(a, b)
$$

for any $a, b \in[0,1]$.

Definition 1.2 A function $F(t):(-\infty,+\infty) \rightarrow[0,1]$ is called a distribution function if it is nondecreasing, left-continuous and $\lim _{t \rightarrow-\infty} F(t)=0$. In addition, if $F(0)=0$, then $F$ is called a distance distribution function.

Definition 1.3 A distance distribution function $F$ satisfying $\lim _{t \rightarrow+\infty} F(t)=1$ is called a Menger distance distribution function. The set of all Menger distance distribution functions is denoted by $D^{+}$. A special Menger distance distribution function is given by

$$
H(t)= \begin{cases}0, & t \leq 0, \\ 1, & t>0 .\end{cases}
$$

Definition 1.4 A probabilistic metric space is a pair $(E, F)$, where $E$ is a nonempty set, $F$ is a mapping from $E \times E$ into $D^{+}$such that if $F_{x, y}$ denotes the value of $F$ at the pair $(x, y)$, the following conditions hold:

(PM-1) $F_{x, y}(t)=H(t)$ if and only if $x=y$;

(PM-2) $F_{x, y}(t)=F_{y, x}(t)$ for all $x, y \in E$ and $t \in(-\infty,+\infty)$;

(PM-3) $F_{x, z}(t)=1, F_{z, y}(s)=1$ implies $F_{x, y}(t+s)=1$ for all $x, y, z \in E$ and $-\infty<t, s<+\infty$.

Definition 1.5 A Menger probabilistic metric space is a triple $(E, F, \triangle)$, where $E$ is a nonempty set, $\triangle$ is a continuous $t$-norm and $F$ is a mapping from $E \times E$ into $D^{+}$such that, if $F_{x, y}$ denotes the value of $F$ at the pair $(x, y)$, the following conditions hold:

(MPM-1) $F_{x, y}(t)=H(t)$ if and only if $x=y$;

(MPM-2) $F_{x, y}(t)=F_{y, x}(t)$ for all $x, y \in E$ and $t \in(-\infty,+\infty)$;

(MPM-3) $F_{x, y}(t+s) \geq \triangle\left(F_{x, z}(t), F_{z, y}(s)\right)$ for all $x, y, z \in E$ and $t>0, s>0$.

In 2014 [18], Su and Zhang gave a new definition of probabilistic metric space, the so-called $S$-probabilistic metric space. In this definition, the triangle inequality has been changed to a new form. 
Definition 1.6 ([18]) An $S$-probabilistic metric space is a pair $(E, F)$, where $E$ is a nonempty set, $F$ is a mapping from $E \times E$ into $D^{+}$such that, if $F_{x, y}$ denotes the value of $F$ at the pair $(x, y)$, the following conditions hold:

$(\mathrm{SPM}-1) F_{x, y}(t)=H(t)$ if and only if $x=y$;

$(\mathrm{SPM}-2) F_{x, y}(t)=F_{y, x}(t)$ for all $x, y \in E$ and $t \in(-\infty,+\infty)$;

(SPM-3) $F_{x, y}(t) \geq F_{x, z}(t) * F_{z, y}(t), \forall x, y, z \in E$, where $F_{x, z}(t) * F_{z, y}(t)$ is the convolution between $F_{x, z}(t)$ and $F_{z, y}(t)$ defined by

$$
F_{x, z}(t) * F_{z, y}(t)=\int_{0}^{+\infty} F_{x, z}(t-u) d F_{z, y}(u)
$$

Example 1.7 ([18]) Let $E$ be a nonempty set, $S$ be a measurable space which consists of some metrics on $E,(\Omega, P)$ be a complete probabilistic measure space and $f: \Omega \rightarrow S$ be a measurable mapping. It is easy to think that $S$ is a random metric on $E$, of course, $(E, S)$ is a random metric space. The following expression of distribution functions $F_{x, y}(t), F_{x, z}(t)$ and $F_{z, y}(t)$ is reasonable:

$$
F_{x, y}(t)=P\left\{f^{-1}\{d \in S ; d(x, y)<t\}\right\}
$$

and

$$
F_{x, z}(t)=P\left\{f^{-1}\{d \in S ; d(x, z)<t\}\right\},
$$

and

$$
F_{z, y}(t)=P\left\{f^{-1}\{d \in S ; d(z, y)<t\}\right\}
$$

for all $x, y, z \in E$. Since

$$
P\left\{f^{-1}\{d \in S ; d(x, y)<t\}\right\} \geq P\left\{f^{-1}\{d \in S ; d(x, z)+d(z, y)<t\}\right\}
$$

and it follows from probabilistic theory that

$$
P\left\{f^{-1}\{d \in S ; d(x, z)+d(z, y)<t\}\right\}=F_{x, z}(t) * F_{z, y}(t) .
$$

Therefore

$$
F_{x, y}(t) \geq F_{x, z}(t) * F_{z, y}(t), \quad \forall x, y, z \in E .
$$

In addition, the conditions (SPM-1), (SPM-2) are obvious.

Definition 1.8 ([7]) A probabilistic normed space is a pair $(E, F)$, where $E$ is a linear space, $F$ is a mapping from $E$ into $D^{+}$such that, if $F_{x}$ denotes the value of $F$ at the pair $x$, the following conditions hold:

(PN-1) $F_{x}(t)=H(t)$ if and only if $x=\theta$;

(PN-2) $F_{\lambda x}(t)=F_{x}\left(\frac{t}{|\lambda|}\right)$ for all $x \in E$ and $t \in(-\infty,+\infty), \lambda \neq 0$;

(PN-3) $F_{x}(t)=1, F_{y}(s)=1$ implies $F_{x+y}(t+s)=1$ for all $x, y \in E$. 
Definition 1.9 ([8]) A Menger probabilistic normed space is a triple $(E, F, \triangle)$, where $E$ is a linear space, $\Delta$ is a continuous $t$-norm and $F$ is a mapping from $E$ into $D^{+}$such that, if $F_{x}$ denotes the value of $F$ at the point $x$, the following conditions hold:

(MPN-1) $F_{x}(t)=H(t)$ if and only if $x=\theta$;

(MPN-2) $F_{\lambda x}(t)=F_{x}\left(\frac{t}{|\lambda|}\right)$ for all $x \in E$ and $t \in(-\infty,+\infty), \lambda \neq 0$;

(MPN-3) $F_{x+y}(t+s) \geq \triangle\left(F_{x}(t), F_{y}(s)\right)$ for all $x, y \in E$ and $t>0, s>0$.

Inspired by reference [18], in this paper, we shall give a new definition of probabilistic normed space, the so-called $S$-probabilistic normed space. In this definition, the triangle inequality has been changed to a new form. We shall also give an example of $S$-probabilistic normed space.

Definition 1.10 An $S$-probabilistic normed space is a pair $(E, F)$, where $E$ is a linear space and $F$ is a mapping from $E$ into $D^{+}$such that, if $F_{x}$ denotes the value of $F$ at the point $x$, the following conditions hold:

$(\mathrm{SPN}-1) F_{x}(t)=H(t)$ if and only if $x=\theta$;

(SPN-2) $F_{\lambda x}(t)=F_{x}\left(\frac{t}{|\lambda|}\right)$ for all $x \in E$ and $t \in(-\infty,+\infty), \lambda \neq 0$;

(SPM-3) $F_{x+y}(t) \geq F_{x}(t) * F_{y}(t), \forall x, y \in E$, where $F_{x}(t) * F_{y}(t)$ is the convolution between $F_{x}(t)$ and $F_{y}(t)$ defined by

$$
F_{x}(t) * F_{y}(t)=\int_{0}^{+\infty} F_{x}(t-u) d F_{y}(u)
$$

Example 1.11 Let $E$ be a linear space, $S$ be a measurable space which consists of some norms on $E,(\Omega, P)$ be a complete probabilistic measure space and $f: \Omega \rightarrow S$ be a measurable mapping. It is easy to think that $S$ is a random metric on $E$, of course, $(E, S)$ is a random normed space. The following expression of distribution functions $F_{x}(t)$ and $F_{y}(t)$ is reasonable:

$$
F_{x}(t)=P\left\{f^{-1}\{\|\cdot\| \in S ;\|x\|<t\}\right\},
$$

and

$$
F_{y}(t)=P\left\{f^{-1}\{\|\cdot\| \in S ;\|y\|<t\}\right\}
$$

for all $x, y \in E$. Since

$$
P\left\{f^{-1}\{\|\cdot\| \in S ;\|x+y\|<t\}\right\} \geq P\left\{f^{-1}\{\|\cdot\| \in S ;\|x\|+\|y\|<t\}\right\},
$$

and it follows from probabilistic theory that

$$
P\left\{f^{-1}\{\|\cdot\| \in S ;\|x\|+\|y\|<t\}\right\}=F_{x}(t) * F_{y}(t) .
$$

Therefore

$$
F_{x+y}(t) \geq F_{x}(t) * F_{y}(t), \quad \forall x, y \in E .
$$

In addition, the conditions (SPN-1), (SPN-2) are obvious. 
Definition $1.12([8])$ Let $(E, F)$ be a probabilistic normed space.

(1) A sequence $\left\{x_{n}\right\}$ in $E$ is said to converge to $x \in E$ if for any given $\varepsilon>0$ and $\lambda>0$, there must exist a positive integer $N=N(\varepsilon, \lambda)$ such that $F_{x_{n}-x}(\varepsilon)>1-\lambda$ whenever $n>N$.

(2) A sequence $\left\{x_{n}\right\}$ in $E$ is called a Cauchy sequence if for any $\varepsilon>0$ and $\lambda>0$, there must exist a positive integer $N=N(\varepsilon, \lambda)$ such that $F_{x_{n}-x_{m}}(\varepsilon)>1-\lambda$, whenever $n, m>N$.

(3) $(E, F, \triangle)$ is said to be complete if each Cauchy sequence in $E$ converges to some point in $E$.

The purpose of this paper is to present a new class of probabilistic normed spaces and to study fixed point problems in this class of probabilistic normed spaces. This paper includes the following two contents: (1) The definition of a new class of probabilistic normed spaces, the so-called $S$-probabilistic normed spaces, is given. In order to study the fixed point problems, some relevant properties of $S$-probabilistic normed spaces are discussed and some basic useful results are obtained; (2) The notion of probabilistic weak convergence is firstly presented in this paper. Therefore the probabilistic weak and strong convergence theorems of fixed points for nonexpansive mappings, asymptotically nonexpansive mappings and strongly pseudocontractive mappings are also proved by using the new methods and techniques.

\section{Basic properties of S-probabilistic normed spaces}

Let $(E, F)$ be an $S$-probabilistic normed space. For any $x \in E$, we define

$$
\|x\|_{F}=\int_{0}^{+\infty} t d F_{x}(t)
$$

Since $t$ is a continuous function and $F_{x}(t)$ is a bounded variation function, so the above integral is well defined. In fact, the above integral is just the mathematical expectation of $F_{x}(t)$. Throughout this paper we assume that

$$
\|x\|_{F}=\int_{0}^{+\infty} t d F_{x}(t)<+\infty, \quad \forall x \in E,
$$

for all probabilistic normed spaces $(E, F)$ presented in this paper.

Next we give the following basic notations of average convergence. Let $(E, F)$ be an $S$ probabilistic normed space:

(1) A sequence $\left\{x_{n}\right\}$ in $E$ is said to converge averagely to $x \in E$ if

$$
\lim _{n \rightarrow \infty} \int_{0}^{+\infty} t d F_{x_{n}-x}(t)=0 .
$$

(2) A sequence $\left\{x_{n}\right\}$ in $E$ is called an average Cauchy sequence if

$$
\lim _{n, m \rightarrow \infty} \int_{0}^{+\infty} t d F_{x_{n}-x_{m}}(t)=0 .
$$

(3) $(E, F)$ is said to be average complete if each average Cauchy sequence in $E$ converges averagely to some point in $E$. 
We denote by $x_{n} \rightarrow x$ that $\left\{x_{n}\right\}$ converges to $x$. It is easy to see that $x_{n} \rightarrow x$ if and only if $\lim _{n \rightarrow \infty} F_{x_{n}-x}(t)=H(t)$ for any given $t \geq 0$. We denote by $x_{n} \Rightarrow x$ that $\left\{x_{n}\right\}$ converges averagely to $x$.

Theorem 2.1 Let $(E, F)$ be an S-probabilistic normed space. For any $x \in E$, we define

$$
\|x\|_{F}=\int_{0}^{+\infty} t d F_{x}(t), \quad \forall x \in E
$$

Then $\|\cdot\|_{N}$ is a norm on $E$.

Proof Since $F_{x}(t)=H(t)(\forall t \geq 0)$, if and only if $x=\theta$, and

$$
\int_{0}^{+\infty} t d H(t)=0
$$

we know the condition $\|x\|_{F}=0 \Leftrightarrow x=y$ holds. For any real number $\lambda$, if $\lambda \neq 0$, we have

$$
\begin{aligned}
\|\lambda x\|_{F} & =\int_{0}^{+\infty} t d F_{\lambda x}(t) \\
& =\int_{0}^{+\infty} t d F_{x}\left(\frac{t}{|\lambda|}\right) \\
& =|\lambda| \int_{0}^{+\infty} \frac{t}{|\lambda|} d F_{x}\left(\frac{t}{|\lambda|}\right) \\
& =|\lambda|\|x\|_{F},
\end{aligned}
$$

if $\lambda=0$, the equality $\|\lambda x\|_{F}=|\lambda|\|x\|_{F}$ is obvious. Next we will prove the triangle inequality. For any $x, y \in E$, from (SFN-5) we have

$$
F_{x+y}(t) \geq \int_{0}^{t} F_{x}(t-u) d F_{y}(u)
$$

By using the property of convolution (integer property), we know

$$
\int_{0}^{+\infty} t d F_{x+y}(t) \leq \int_{0}^{+\infty} t d F_{x}(t)+\int_{0}^{+\infty} t d F_{y}(t)
$$

which implies

$$
\|x+y\|_{F} \leq\|x\|_{F}+\|y\|_{F} .
$$

This completes the proof.

Definition 2.2 A $b$-normed space is a pair $(E,\|\cdot\|)$, where $E$ is a linear space, $\|\cdot\|: E \rightarrow$ $R^{+}=[0,+\infty)$ is a real function and $b \geq 1$ is a constant, the following conditions hold:

(BN-1) $\|x\|=0 \Leftrightarrow x=\theta$;

(BN-2) $\|\lambda x\|=|\lambda|\|x\|$ for all $x \in E, \lambda \in(-\infty,+\infty)$;

(BN-3) $\|x+y\| \leq b\|x\|+b\|y\|$ for all $x, y \in E$. 
Theorem 2.3 Let $\left(E, B, \triangle_{1}\right)$ be a Menger probabilistic normed space. For any $x \in E$, we define

$$
\|x\|_{B}=\int_{0}^{+\infty} t d F_{x}(t)
$$

Then $\|\cdot\|_{B}$ is a $b$-norm with $b=2$ on $E$.

Proof The conditions (BN-1) and (BN-2) are obvious. We only need to prove the general triangle inequality (BN-3)

$$
\|x+y\|_{B} \leq 2\|x\|_{B}+2\|y\|_{B}, \quad \forall x, y \in E
$$

In fact,

$$
\begin{aligned}
\|x+y\|_{B} & =\int_{0}^{+\infty} t d F_{x+y}(t) \\
& =\int_{0}^{+\infty} t d \max \left\{F_{x}\left(\frac{t}{2}\right)+F_{y}\left(\frac{t}{2}\right)-1,0\right) \\
& =\int_{0}^{+\infty} t d\left(F_{x}\left(\frac{t}{2}\right)+F_{y}\left(\frac{t}{2}\right)\right) \\
& =\int_{0}^{+\infty} t d F_{x}\left(\frac{t}{2}\right)+\int_{0}^{+\infty} t d F_{y}\left(\frac{t}{2}\right) \\
& =2 \int_{0}^{+\infty} \frac{t}{2} d F_{x}\left(\frac{t}{2}\right)+2 \int_{0}^{+\infty} \frac{t}{2} d F_{y}\left(\frac{t}{2}\right) \\
& =2\|x\|_{B}+2\|y\|_{B} .
\end{aligned}
$$

This completes the proof.

Definition 2.4 Let $(E, F)$ be an $S$-probabilistic normed space. Let $f$ be a real linear functional defined on $E$, if $x_{n} \rightarrow x$ implies $f\left(x_{n}\right) \rightarrow f(x), f$ is said to be a continuous linear functional. Let $E^{*_{1}}$ denote the set of all linear continuous functionals defined on $E$. $E^{*_{1}}$ is called the first-type conjugate space of $E$. If $x_{n} \Rightarrow x$ implies $f\left(x_{n}\right) \rightarrow f(x), f$ is said to be an average continuous linear functional. Let $E^{*_{2}}$ denote the set of all linear average continuous functionals defined on $E . E^{*_{2}}$ is called the second-type conjugate space of $E$.

Theorem $2.5 E^{*_{1}} \subset E^{*_{2}}$.

Proof We need only to prove that $x_{n} \Rightarrow x$ implies $x_{n} \rightarrow x$ as $n \rightarrow \infty$. If not,

$$
\left\|x_{n}-x\right\|_{B}=\int_{0}^{+\infty} t d F_{x_{n}-x}(t) \rightarrow 0
$$

as $n \rightarrow \infty$, but

$$
\lim _{n \rightarrow \infty} F_{x_{n}-x}(t)=H(t)
$$


is not right. Then there exist numbers $t_{0}>0,0<\lambda_{0}<1$ and a subsequence $\left\{n_{k}\right\}$ of $\{n\}$ such that $F_{x_{n_{k}}-x}\left(t_{0}\right) \leq \lambda_{0}$ for all $k \geq 1$. In this case, we have

$$
\begin{aligned}
\left\|x_{n_{k}}-x\right\|_{B} & =\int_{0}^{+\infty} t d F_{x_{n_{k}}-x}(t) \\
& =\int_{0}^{t_{0}} t d F_{x_{n_{k}}-x}(t)+\int_{t_{0}}^{+\infty} t d F_{x_{n_{k}-x}}(t) \\
& \geq \int_{t_{0}}^{+\infty} t d F_{x_{n_{k}}-x}(t) \geq t_{0}\left(1-F_{x_{n_{k}-x}}(t)\right) \\
& \geq t_{0}\left(1-\lambda_{0}\right)>0 .
\end{aligned}
$$

This is a contradiction. This completes the proof.

Definition 2.6 Let $(E, F)$ be an $S$-probabilistic normed space. A sequence $\left\{x_{n}\right\} \subset E$ is said to probabilistically converge weakly to an element $x$ if $\lim _{n \rightarrow \infty} f\left(x_{n}\right)=f(x)$ for any $f \in E^{* 1}$.

\section{Weak and strong convergence theorems for some nonlinear mappings in $S$-probabilistic spaces}

Theorem 3.1 Let $(E, F)$ be an S-probabilistic normed (Banach) space. Then $\left(E,\|\cdot\|_{F}\right)$ is an inner product (Hilbert) space if and only if

$$
\int_{0}^{+\infty} t^{2} d\left(2 F_{x}(t)+2 F_{y}(t)-F_{x+y}-F_{x-y}(t)\right)=0
$$

for all $x, y \in E$.

Proof Condition (3.1) can be rewritten to

$$
2 \int_{0}^{+\infty} t^{2} d F_{x}(t)+2 \int_{0}^{+\infty} t^{2} d F_{x}(t)=\int_{0}^{+\infty} t^{2} d F_{x+y}(t)+\int_{0}^{+\infty} t^{2} d F_{x-y}(t) .
$$

By using the probabilistic theory, we know that (3.2) is equivalent to

$$
2\left(\int_{0}^{+\infty} t d F_{x}(t)\right)^{2}+2\left(\int_{0}^{+\infty} t d F_{x}(t)\right)^{2}=\left(\int_{0}^{+\infty} t d F_{x+y}(t)\right)^{2}+\left(\int_{0}^{+\infty} t d F_{x-y}(t)\right)^{2} .
$$

Therefore condition (3.1) is equivalent to

$$
2\|x\|_{F}^{2}+2\|y\|_{F}^{2}=\|x+y\|_{F}^{2}+\|x-y\|_{F}^{2}
$$

It is well known that (3.3) is called the parallelogram condition for the inner product space, so that the conclusion of Theorem 3.1 is right. The proof is complete.

Theorem 3.2 Let (E,F) be an S-probabilistic normed (Banach) space which satisfies condition (3.1). Then

$$
\langle x, y\rangle=\frac{1}{4} \int_{0}^{+\infty} t^{2} d\left(F_{x+y}(t)-F_{x-y}(t)\right)
$$


for all $x, y \in E$ is an inner product and

$$
\|x\|_{F}=\sqrt{\langle x, x\rangle}
$$

for all $x \in E$.

Proof By using Theorem 3.1, we know if condition (3.1) holds, then $\left(E,\|\cdot\|_{F}\right)$ satisfies the parallelogram condition (3.3). In this case, we know that

$$
\langle x, y\rangle=\frac{1}{4}\left(\|x+y\|_{F}^{2}-\|x-y\|_{F}^{2}\right)
$$

for all $x, y \in E$ is an inner product. On the other hand, (3.4) can be rewritten to

$$
\langle x, y\rangle=\frac{1}{4}\left(\left(\int_{0}^{+\infty} t d F_{x+y}(t)\right)^{2}-\left(\int_{0}^{+\infty} t d F_{x-y}(t)\right)^{2}\right)
$$

for all $x, y \in E$. By using the probabilistic theory, we know that

$$
\left(\int_{0}^{+\infty} t d F_{x+y}(t)\right)^{2}-\left(\int_{0}^{+\infty} t d F_{x-y}(t)\right)^{2}=\int_{0}^{+\infty} t^{2} d F_{x+y}(t)-\int_{0}^{+\infty} t^{2} d F_{x-y}(t) .
$$

This implies that

$$
\left(\int_{0}^{+\infty} t d F_{x+y}(t)\right)^{2}-\left(\int_{0}^{+\infty} t d F_{x-y}(t)\right)^{2}=\int_{0}^{+\infty} t^{2} d\left(F_{x+y}(t)-F_{x-y}(t)\right) .
$$

Therefore

$$
\langle x, y\rangle=\frac{1}{4} \int_{0}^{+\infty} t^{2} d\left(F_{x+y}(t)-F_{x-y}(t)\right)
$$

for all $x, y \in E$ is an inner product. From (3.4), the second conclusion is obvious. The proof is complete.

Definition 3.3 Let $(E, F)$ be a probabilistic normed space. A mapping $T: E \rightarrow E$ is said to be Lipschitz if there exists a constant $L \geq 1$ such that

$$
F_{T x-T y}(t) \geq F_{x-y}\left(\frac{t}{L}\right), \quad \forall t \in(-\infty,+\infty)
$$

for all $x, y \in E$, if $L=1$, the mapping $T$ is said to be nonexpansive.

Theorem 3.4 Let $(E, F)$ be a probabilistic Banach space with condition (3.1), $T: E \rightarrow E$ be a nonexpansive mapping with nonempty fixed point set $F(T)$. For any guess $x_{0} \in E$, the Mann iterative sequence $\left\{x_{n}\right\}$ is defined by

$$
x_{n+1}=\left(1-\alpha_{n}\right) x_{n}+\alpha_{n} T x_{n},
$$


where $\left\{\alpha_{n}\right\}$ is a real number sequence which was assumed to satisfy the condition

$$
\sum_{n=0}^{\infty} \alpha_{n}\left(1-\alpha_{n}\right)=\infty
$$

Then $\left\{x_{n}\right\}$ probabilistically converges weakly to a fixed point of $T$.

Proof From Definition 3.3, we have

$$
\begin{aligned}
\|T x-T y\|_{F} & =\int_{-\infty}^{+\infty} t d F_{T x-T y}(t) \\
& \leq \int_{-\infty}^{+\infty} t d F_{x-y}(t)=\|x-y\|_{F}, \quad \forall x, y \in E,
\end{aligned}
$$

so that $T$ is also a nonexpansive mapping in the framework of Banach space $\left(E,\|\cdot\|_{F}\right)$. Therefore, by using Mann's result [15], we know that $\left\{x_{n}\right\}$ converges weakly to a fixed point $x^{*}$ of $T$. That is, for any $f \in\left(E,\|\cdot\|_{F}\right)^{*}$, we have

$$
\lim _{n \rightarrow \infty} f\left(x_{n}\right)=f\left(x^{*}\right)
$$

By using Theorem 2.5, we know that $(E, F)^{*} \subseteq\left(E,\|\cdot\|_{F}\right)^{*}$, so for any $f \in(E, F)^{*}$, we have also that

$$
\lim _{n \rightarrow \infty} f\left(x_{n}\right)=f\left(x^{*}\right)
$$

That is, the Mann iterative sequence $\left\{x_{n}\right\}$ probabilistically converges weakly to $x^{*}$. The proof is complete.

In 2004, Xu proved the following strong convergence theorem for nonexpansive mappings in a uniformly smooth Banach space.

Theorem 3.5 ([16]) Let (E,F) be a uniformly smooth Banach space, $C$ be a closed convex subset of $E$ and $T: C \rightarrow C$ be a nonexpansive mapping with nonempty fixed point set and $f: C \rightarrow C$ be a contraction. Assume that the sequence $\left\{\alpha_{n}\right\} \subset[0,1]$ satisfies the following conditions:

(1) $\lim _{n \rightarrow \infty} \alpha_{n}=0$;

(2) $\sum_{n=1}^{\infty} \alpha_{n}=+\infty$;

(3) either $\lim _{n \rightarrow \infty} \frac{\alpha_{n+1}}{\alpha_{n}}=1$ or $\sum_{n=1}^{\infty}\left|\alpha_{n+1}-\alpha_{n}\right|=+\infty$.

For any guess $x_{0} \in E$, the iterative sequence $\left\{x_{n}\right\}$ generated by

$$
x_{n+1}=+\alpha_{n} f\left(x_{n}\right)+\left(1-\alpha_{n}\right) T x_{n}
$$

converges strongly to a fixed point of $T$.

By using Theorem 3.5 and an ingenious method, we can prove the following convergence theorem for nonexpansive mappings in the framework of probabilistic Banach space. 
Theorem 3.6 Let (E,F) be a probabilistic Banach space with condition (3.1), $T: E \rightarrow E$ be a nonexpansive mapping with nonempty fixed point set $F(T)$ and $f: C \rightarrow C$ be a contraction. Assume that the sequence $\left\{\alpha_{n}\right\} \subset[0,1]$ satisfies the following conditions:

(1) $\lim _{n \rightarrow \infty} \alpha_{n}=0$;

(2) $\sum_{n=1}^{\infty} \alpha_{n}=+\infty$;

(3) either $\lim _{n \rightarrow \infty} \frac{\alpha_{n+1}}{\alpha_{n}}=1$ or $\sum_{n=1}^{\infty}\left|\alpha_{n+1}-\alpha_{n}\right|=+\infty$.

For any guess $x_{0} \in E$, the iterative sequence $\left\{x_{n}\right\}$ generated by

$$
x_{n+1}=+\alpha_{n} f\left(x_{n}\right)+\left(1-\alpha_{n}\right) T x_{n}
$$

probabilistically converges to a fixed point of $T$.

Proof By the same reasoning as in Theorem 3.4, we know that the mapping $T: E \rightarrow E$ is also a nonexpansive mapping in the framework of Banach space $\left(E,\|\cdot\|_{F}\right)$. Condition (3.1) implies that $\left(E,\|\cdot\|_{F}\right)$ is a Hilbert space, so the conditions of Theorem 3.5 were satisfied. By using Theorem 3.5, we know that the iterative sequence $\left\{x_{n}\right\}$ converges in norm $\|\cdot\|_{F}$ to a fixed point $x^{*}$ of $T$. That is, $x_{n} \Rightarrow x^{*}$, which implies $x_{n} \rightarrow x^{*}$ (see the proof of Theorem 2.5). The proof is complete.

Definition 3.7 Let $(E, F)$ be a probabilistic normed space. A mapping $T: E \rightarrow E$ is said to be probabilistic asymptotically nonexpansive if

$$
F_{T^{n} x-T^{n} y}(t) \geq F_{x-y}\left(\frac{t}{k_{n}}\right), \quad \forall t \in(-\infty,+\infty)
$$

for all $x, y \in E$ and all positive integers $n$, where $k_{n} \in[1,+\infty)$ and $\lim _{n \rightarrow \infty} k_{n}=1$.

In 2000, Osilike and Aniagbosor proved the following weak convergence theorem for asymptotically nonexpansive mappings in the framework of uniformly convex Banach space with Opial's condition.

Theorem 3.8 ([17]) Let E be a uniformly convex Banach space satisfying Opial's condition, and let $K$ be a nonempty closed convex subset of $E$. Let $T: K \rightarrow K$ be an asymptotically nonexpansive mapping with $F(T) \neq \emptyset$ and a sequence $\left\{k_{n}\right\} \subset[1,+\infty)$ such that $\lim k_{n}=1$ and $\sum_{n=1}^{\infty}\left(k_{n}-1\right)<+\infty$. Let $\left\{u_{n}\right\}$ and $\left\{v_{n}\right\}$ be bounded sequences in $K$, and let $\left\{a_{n}\right\},\left\{b_{n}\right\}$, $\left\{c_{n}\right\},\left\{\alpha_{n}\right\},\left\{\beta_{n}\right\},\left\{\gamma_{n}\right\}$ be real sequences in $[0,1)$ satisfying the conditions:

(1) $a_{n}+b_{n}+c_{n}=\alpha_{n}+\beta_{n}+\gamma_{n}=1, \forall n \geq 1$

(2) $0<a \leq \beta_{n} \leq b<1, \forall n \geq 1$;

(3) $\lim _{n \rightarrow \infty} \beta_{n}=0$;

(4) $\sum_{n=1}^{\infty} c_{n}<+\infty, \sum_{n=1}^{\infty} \gamma_{n}<+\infty$.

Then the sequence generated from an arbitrary $x_{1} \in K$ by

$$
\begin{aligned}
& y_{n}=a_{n} x_{n}+b_{n} T^{n} x_{n}+c_{n} u_{n}, \\
& x_{n+1}=\alpha_{n} x_{n}+\beta_{n} T^{n} y_{n}+c_{n} v_{n},
\end{aligned}
$$

converges weakly to some fixed point of $T$. 
By using Theorem 3.8 and an ingenious method as in Theorem 3.6, we can prove the following probabilistic weak convergence theorem for nonexpansive mappings in the framework of probabilistic Banach space.

Theorem 3.9 Let $(E, F)$ be a probabilistic Banach space with condition (3.1). Let $T: E \rightarrow E$ be an asymptotically nonexpansive mapping with $F(T) \neq \emptyset$ and a sequence $\left\{k_{n}\right\} \subset[1,+\infty)$ such that $\lim k_{n}=1$ and $\sum_{n=1}^{\infty}\left(k_{n}-1\right)<+\infty$. Let $\left\{u_{n}\right\}$ and $\left\{v_{n}\right\}$ be bounded sequences in $K$, and let $\left\{a_{n}\right\},\left\{b_{n}\right\},\left\{c_{n}\right\},\left\{\alpha_{n}\right\},\left\{\beta_{n}\right\},\left\{\gamma_{n}\right\}$ be real sequences in $[0,1)$ satisfying the conditions:

(1) $a_{n}+b_{n}+c_{n}=\alpha_{n}+\beta_{n}+\gamma_{n}=1, \forall n \geq 1$;

(2) $0<a \leq \beta_{n} \leq b<1, \forall n \geq 1$;

(3) $\lim _{n \rightarrow \infty} \beta_{n}=0$;

(4) $\sum_{n=1}^{\infty} c_{n}<+\infty, \sum_{n=1}^{\infty} \gamma_{n}<+\infty$

Then the sequence generated from an arbitrary $x_{1} \in K$ by

$$
\begin{aligned}
& y_{n}=a_{n} x_{n}+b_{n} T^{n} x_{n}+c_{n} u_{n}, \\
& x_{n+1}=\alpha_{n} x_{n}+\beta_{n} T^{n} y_{n}+c_{n} v_{n},
\end{aligned}
$$

probabilistically converges weakly to some fixed point of $T$.

Proof Condition (3.1) implies that $\left(E,\|\cdot\|_{F}\right)$ is a Hilbert space. Since $T: E \rightarrow E$ is probabilistic asymptotically nonexpansive, from Definition 3.7 we have

$$
\int_{0}^{+\infty} t d F_{T^{n} x-T^{n} y}(t) \leq \int_{0}^{+\infty} t d F_{x-y}\left(\frac{t}{k_{n}}\right)
$$

for all $x, y \in E$ and all positive integers $n$, where $k_{n} \in[1,+\infty)$ and $\lim _{n \rightarrow \infty} k_{n}=1$. This implies that

$$
\left\|T^{n} x-T^{n} y\right\|_{F} \leq k_{n}\|x-y\|_{F}
$$

for all $x, y \in E$ and all positive integers $n$, where $k_{n} \in[1,+\infty)$ and $\lim _{n \rightarrow \infty} k_{n}=1$. Then $T$ is an asymptotically nonexpansive mapping from a Hilbert space $\left(E,\|\cdot\|_{F}\right)$ into itself. By using Theorem 3.8, we know that the iterative sequence $\left\{x_{n}\right\}$ converges weakly to some fixed point $x^{*}$ of $T$. That is, for any $f \in\left(E,\|\cdot\|_{F}\right)^{*}$, we have $\lim _{n \rightarrow \infty} f\left(x_{n}\right)=f\left(x^{*}\right)$. By using Theorem 2.5, we know that $(E, F)^{*} \subset\left(E,\|\cdot\|_{F}\right)^{*}$; therefore, for any $f \in(E, F)^{*}$, we have $\lim _{n \rightarrow \infty} f\left(x_{n}\right)=f\left(x^{*}\right)$. Then $\left\{x_{n}\right\}$ probabilistically converges weakly to some fixed point of $T$. The proof is complete.

Let $E$ be a real Banach space and let $J$ denote the normalized duality mapping from $E$ into $E^{*}$ given by

$$
J(x)=\left\{f \in E^{*}:\langle x, f\rangle=\|f\|^{2}=\|x\|^{2}\right\}, \quad x \in E .
$$

Recall that a mapping $T$ with domain $D(T)$ and range $R(T)$ in a Banach space is said to be pseudocontractive if there exists $j(x-y) \in J(x-y)$ such that

$$
\langle T x-T y, j(x-y)\rangle \leq\|x-y\|^{2}, \quad \forall x, y \in D(T) .
$$


Recall that a mapping with domain $D(T)$ and range $R(T)$ in a Banach space is said to be strongly pseudocontractive ( $k$-strongly pseudocontractive) if there exists $j(x-y) \in J(x-y)$ such that

$$
\langle T x-T y, j(x-y)| \leq k\|x-y\|^{2}, \quad \forall x, y \in D(T),
$$

where $k \in(0,1)$ is a constant. In a Hilbert space, the above inequality can be written as

$$
\langle T x-T y, x-y\rangle \leq k\|x-y\|^{2}, \quad \forall x, y \in D(T) .
$$

In 1974, Deimling [19] proved the following fixed point theorem.

Theorem 3.10 Let $E$ be a real Banach space, $K$ be a nonempty closed convex subset of $E$, and $T: K \rightarrow K$ be a continuous strongly pseudocontractive mapping. Then $T$ has a unique fixed point in $K$.

Lemma 3.11 Let $E$ be a real Hilbert space, $K$ be a nonempty closed convex subset of $E$, and $T: K \rightarrow K$ be an L-Lipschitz $k$-strongly pseudocontractive mapping with Lipschitz constant $L \geq 1$. Then $T$ has a unique fixed point $x^{*}$ in $K$ and for any given $x_{0} \in K$, the iterative sequence

$$
x_{n+1}=\left(1-\mu_{n}\right) x_{n}+\mu_{n} T x_{n}
$$

converges strongly to $x^{*}$, where $\left\{\mu_{n}\right\} \subset(0,1)$ is a sequence of real numbers which satisfies the following condition:

$$
0<\mu_{n}<\mu<\frac{2-2 k}{L^{2}+1-2 k}, \quad \forall n \geq 1
$$

for some constant $\mu$.

Proof From condition (3.6), we have

$$
\begin{aligned}
0 & <\mu<\frac{2-2 k}{L^{2}+1-2 k} \\
& \Leftrightarrow \quad \mu\left(1+L^{2}-2 k\right)<2-2 k \\
& \Leftrightarrow \quad \mu\left(1+L^{2}-2 k\right)+2 k<2 \\
& \Leftrightarrow \quad \mu\left(1+L^{2}\right)+2 k-2 \mu k<2 \\
& \Leftrightarrow \quad \mu\left(1+L^{2}\right)+2 k-2 \mu k-2<0 \\
& \Leftrightarrow \quad \mu+\mu L^{2}+2 k-2 \mu k-2<0 \\
& \Leftrightarrow \quad \mu^{2}+\mu^{2} L^{2}+2 \mu k-2 \mu^{2} k-2 \mu<0 \\
& \Leftrightarrow \quad 1+\mu^{2}+\mu^{2} L^{2}+2 \mu k-2 \mu^{2} k-2 \mu<1 \\
& \Leftrightarrow \quad(1-\mu)^{2}+\mu^{2} L^{2}+2 \mu(1-\mu) k<1 .
\end{aligned}
$$


Let $T_{\mu}=(1-\mu) I+\mu T$, where $I$ is the identity operator. For $\forall x, y \in K$, we have

$$
\begin{aligned}
& \left\|T_{\mu} x-T_{\mu} y\right\|^{2} \\
& \quad=\|(1-\mu) x+\mu T x-(1-\mu) y-\mu T y\|^{2} \\
& \quad=\langle(1-\mu) x+\mu T x-(1-\mu) y-\mu T y,(1-\mu) x+\mu T x-(1-\mu) y-\mu T y\rangle \\
& =\langle(1-\mu)(x-y)+\mu(T x-T y),(1-\mu)(x-y)+\mu(T x-T y)\rangle \\
& =(1-\mu)^{2}\|x-y\|^{2}+\mu^{2}\|T x-T y\|^{2}+2 \mu(1-\mu)\langle T x-T y, x-y\rangle \\
& \quad \leq(1-\mu)^{2}\|x-y\|^{2}+\mu^{2} L^{2}\|x-y\|^{2}+2 \mu(1-\mu) k\|x-y\|^{2} \\
& \quad=\left((1-\mu)^{2}+\mu^{2} L^{2}+2 \mu(1-\mu) k\right)\|x-y\|^{2} .
\end{aligned}
$$

From (3.7) we know

$$
0<(1-\mu)^{2}+\mu^{2} L^{2}+2 \mu(1-\mu) k<1
$$

From (3.8) we know that $T_{\mu}$ is a contractive mapping from $K$ into itself. By using the Banach contraction mapping principle, we know that $T_{\mu}$ has a unique fixed point $x^{*}$. That is,

$$
x^{*}=T_{\mu} x^{*}=(1-\mu) x^{*}+\mu T x^{*},
$$

which is equivalent to $x^{*}=T x^{*}$.

From condition (3.6), we have

$$
\begin{aligned}
& \mu_{n}\left(1+L^{2}-2 k\right)<\mu\left(1+L^{2}-2 k\right), \\
& \mu_{n}\left(1+L^{2}\right)+2 k-2 \mu k-2<\mu\left(1+L^{2}\right)+2 k-2 \mu k-2, \\
& \mu_{n}+\mu_{n} L^{2}+2 k-2 \mu_{n} k-2<\mu+\mu L^{2}+2 k-2 \mu k-2, \\
& \mu_{n}^{2}+\mu_{n}^{2} L^{2}+2 \mu_{n} k-2 \mu_{n}^{2} k-2 \mu_{n}<\mu^{2}+\mu^{2} L^{2}+2 \mu k-2 \mu^{2} k-2 \mu<0, \\
& 1+\mu_{n}^{2}+\mu_{n}^{2} L^{2}+2 \mu_{n} k-2 \mu_{n}^{2} k-2 \mu_{n}<1+\mu^{2}+\mu^{2} L^{2}+2 \mu k-2 \mu^{2} k-2 \mu<1, \\
& \left(1-\mu_{n}\right)^{2}+\mu_{n}^{2} L^{2}+2 \mu_{n}\left(1-\mu_{n}\right) k<(1-\mu)^{2}+\mu^{2} L^{2}+2 \mu(1-\mu) k<1 .
\end{aligned}
$$

Next we prove that the iterative sequence defined by (3.5) converges strongly to $x^{*}$. Observe

$$
\begin{aligned}
\left\|x_{n+1}-x^{*}\right\|^{2} \\
\quad=\left\|\left(1-\mu_{n}\right)\left(x_{n}-x^{*}\right)+\mu_{n}\left(T x_{n}-x^{*}\right)\right\|^{2} \\
\quad=\left\langle\left(1-\mu_{n}\right)\left(x_{n}-x^{*}\right)+\mu_{n}\left(T x_{n}-x^{*}\right),\left(1-\mu_{n}\right)\left(x_{n}-x^{*}\right)+\mu_{n}\left(T x_{n}-x^{*}\right)\right\rangle \\
\quad=\left(1-\mu_{n}\right)^{2}\left\|x_{n}-x^{*}\right\|^{2}+\mu_{n}^{2}\left\|T x_{n}-T x^{*}\right\|^{2}+2 \mu_{n}\left(1-\mu_{n}\right)\left\langle T x_{n}-T x^{*}, x_{n}-x^{*}\right\rangle \\
\quad \leq\left(1-\mu_{n}\right)^{2}\left\|x_{n}-x^{*}\right\|^{2}+\mu_{n}^{2} L^{2}\left\|x_{n}-x^{*}\right\|^{2}+2 \mu_{n}\left(1-\mu_{n}\right) k\left\|x_{n}-x^{*}\right\|^{2} \\
\quad=\left(\left(1-\mu_{n}\right)^{2}+\mu_{n}^{2} L^{2}+2 \mu_{n}\left(1-\mu_{n}\right) k\right)\left\|x_{n}-x^{*}\right\|^{2} .
\end{aligned}
$$


From (3.9) and (3.10) we have

$$
\left\|x_{n+1}-x^{*}\right\|^{2} \leq\left((1-\mu)^{2}+\mu^{2} L^{2}+2 \mu(1-\mu) k\right)\left\|x_{n}-x^{*}\right\|^{2} \rightarrow 0
$$

as $n \rightarrow \infty$, so that $x_{n} \rightarrow x^{*}$ as $n \rightarrow \infty$. This completes the proof.

Next, we give the definition of probabilistic strongly pseudocontractive mappings in a probabilistic normed space.

Definition 3.12 Let $(E, F)$ be a probabilistic normed space. A mapping $T: E \rightarrow E$ is said to be probabilistic strongly pseudocontractive ( $k$-strongly pseudocontractive) if

$$
F_{T x-T y+x-y}(\sqrt{t}) \geq 4 k F_{x-y}\left(t^{2}\right)+F_{T x-T y-(x-y)}(\sqrt{t})
$$

for all $x, y \in E, t \in[0,+\infty)$, where $k \in(0,1)$ is a constant.

Theorem 3.13 Let $(E, F)$ be a probabilistic Banach space with condition (3.1), $K$ be a nonempty closed convex subset of $E$, and $T: K \rightarrow K$ be an L-Lipschitz $k$-strongly pseudocontractive mapping with Lipschitz constant $L \geq 1$. Then $T$ has a unique fixed point $x^{*}$ in $K$, and for any given $x_{0} \in K$, the iterative sequence

$$
x_{n+1}=\left(1-\mu_{n}\right) x_{n}+\mu_{n} T x_{n}
$$

probabilistically converges to $x^{*}$, where $\left\{\mu_{n}\right\} \subset(0,1)$ is a sequence of real numbers which satisfies the following condition:

$$
0<\mu_{n}<\mu<\frac{2-2 k}{L^{2}+1-2 k}, \quad \forall n \geq 1,
$$

for some constant $\mu$.

Proof Noting that

$$
F_{T x-T y+x-y}(\sqrt{t}), \quad 4 k F_{x-y}\left(t^{2}\right)+F_{T x-T y-(x-y)}(\sqrt{t})
$$

are nondecreasing and

$$
F_{T x-T y+x-y}(\sqrt{0})=0, \quad 4 k F_{x-y}\left(0^{2}\right)+F_{T x-T y-(x-y)}(\sqrt{0})=0 .
$$

Therefore, from (3.11) we have, for all $x, y \in E$, that

$$
\int_{0}^{+\infty} t d F_{T x-T y+x-y}(\sqrt{t}) \leq \int_{0}^{+\infty} t d\left(4 k F_{x-y}\left(t^{2}\right)+F_{T x-T y-(x-y)}(\sqrt{t})\right)
$$

which implies that

$$
\frac{1}{4} \int_{0}^{+\infty} t^{2} d\left(F_{T x-T y+x-y}(t)-F_{T x-T y+y-x}(t)\right) \leq k \int_{0}^{+\infty} \sqrt{t} d F_{x-y}(t)
$$


for all $x, y \in E$. From Theorem 3.2 and Definition 3.3, we have

$$
\begin{aligned}
& \langle T x-T y, x-y\rangle \leq k\|x-y\|_{F}^{2}, \quad \forall x, y \in E, \\
& \|T x-T y\|_{F} \leq L\|x-y\|_{F}, \quad \forall x, y \in E .
\end{aligned}
$$

This shows that $T$ is an $L$-Lipschitz $k$-strongly pseudocontractive mapping with Lipschitz constant $L \geq 1$ in a Hilbert space $\left(E,\|\cdot\|_{F}\right)$. By using Lemma 3.11, we know that $T$ has a unique fixed point $x^{*}$ in $K$, and for any given $x_{0} \in K$, the iterative sequence defined by (3.12) converges in norm $\|\cdot\|_{F}$ to $x^{*}$. Noting that the convergence in norm $\|\cdot\|_{F}$ implies the probabilistic convergence, so that the iterative sequence defined by (3.12) probabilistically converges to $x^{*}$. This completes the proof.

Remark The research into probabilistic normed spaces and relevant fixed point theory is an important topic. Many relevant results have been given by some authors [14, 20-34]. However, the profound relationship with the probabilistic theory has not received close attention. The $S$-probabilistic normed spaces and relevant probabilistic methods will play an important role in the theory and applications.

Competing interests

The authors declare that they have no competing interests.

Authors' contributions

All authors contributed equally and significantly in writing this article. All authors read and approved the final manuscript.

\section{Author details}

${ }^{1}$ Department of Mathematics, Hebei North University, Zhangjiakou, 075000, China. ${ }^{2}$ Department of Mathematics, Tianjin Polytechnic University, Tianjin, 300387, China.

\section{Acknowledgements}

This project is supported by the major project of Hebei North University under grant No. ZD201304.

Received: 22 May 2015 Accepted: 10 August 2015 Published online: 25 August 2015

\section{References}

1. Menger, K: Statistical metrics. Proc. Natl. Acad. Sci. USA 28, 535-537 (1942)

2. Sehgal, VM: Some fixed point theorems in functional analysis and probability. Ph.D. thesis, Wayne State University, Detroit, MI (1966)

3. Šerstnev, AN: Random normed spaces: problems of completeness. Kazan. Gos. Univ. Ucen. Zap. 122, 3-20 (1962)

4. Šerstnev, AN: On the notion of a random normed space. Dokl. Akad. Nauk SSSR 149(2), 280-283 (1963). English transl. Sov. Math. Dok. 4, 388-390 (1963)

5. Šerstnev, AN: Best approximation problems in random normed spaces. Dokl. Akad. Nauk SSSR 149(3), 539-542 (1963)

6. Šerstnev, AN: On a probabilistic generalization of a metric spaces. Kazan. Gos. Univ. Ucen. Zap. 124, 3-11 (1964)

7. Alsina, C, Schweizer, B, Sklar, A: On the definition of a probabilistic normed space. Aequ. Math. 46, 91-98 (1993)

8. Lafuerza-Guillén, B, Rodríguez-Lallena, JA, Sempi, C: Some classes of probabilistic normed spaces. Rend. Mat. 17, 237-252 (1997)

9. Lafuerza-Guillén, B, Rodríguez-Lallena, JA, Sempi, C: Completion of probabilistic normed spaces. Int. J. Math. Comput. Sci. 18, 649-652 (1995)

10. Lafuerza-Guillén, B, Rodríguez-Lallena, JA, Sempi, C: A study of boundedness in probabilistic normed spaces. J. Math. Anal. Appl. 232, 183-196 (1999)

11. Lafuerza-Guillén, B, Rodríguez-Lallena, JA, Sempi, C: Probabilistic norms for linear operators. J. Math. Anal. Appl. 220 462-476 (1998)

12. Lafuerza-Guillén, B: Latest results on probabilistic normed spaces. Int. J. Math. Anal. 1, 269-309 (2006)

13. Sempi, C: A short and partial history of probabilistic normed spaces. Mediterr. J. Math. 3, 283-300 (2006)

14. Hadzi, O, Pap, E: Fixed Point Theory in Probabilistic Metric Spaces. Kluwer Academic Publishers, Dordrecht (2001)

15. Mann, WR: Mean value method in iteration. Proc. Am. Math. Soc. 4, 506-510 (1953)

16. Xu, HK: Viscosity approximation methods for nonexpansive mappings. J. Math. Anal. Appl. 298, 279-291 (2004)

17. Osilike, M, Aniagbosor, S: Weak and strong convergence theorems for fixed points of asymptotically nonexpansive mappings. Math. Comput. Model. 32,1181-1191 (2000)

18. Su, Y, Zhang, J: Fixed point and best proximity point theorems for contractions in new class of probabilistic metric spaces. Fixed Point Theory Appl. 2014, 170 (2014)

19. Deimling, K: Zeros of accretive operators. Manuscr. Math. 13, 365-374 (1974) 
20. Sehgal, VM, Bharucha-Reid, AT: Fixed points of contraction mappings on PM-spaces. Math. Syst. Theory 6, 97-102 (1972)

21. Cho, YJ, Park, KS, Chang, SS: Fixed point theorems in metric spaces and probabilistic metric spaces. Int. J. Math. Math. Sci. 19, 243-252 (1996)

22. Čirič, LB: Some new results for Banach contractions and Edelstein contractive mappings on fuzzy metric spaces. Chaos Solitons Fractals 42, 146-154 (2009)

23. Čirič, LB, Mihet, D, Saadati, R: Monotone generalized contractions in partially ordered probabilistic metric spaces. Topol. Appl. 156, 2838-2844 (2009)

24. Fang, JE: Common fixed point theorems of compatible and weakly compatible maps in Menger spaces. Nonlinear Anal. 71, 1833-1843 (2009)

25. Kamran, T: Common fixed points theorems for fuzzy mappings. Chaos Solitons Fractals 38, 1378-1382 (2008)

26. Liu, Y, Li, Z: Coincidence point theorems in probabilistic and fuzzy metric spaces. Fuzzy Sets Syst. 158, 58-70 (2007)

27. Mihet, D: Altering distances in probabilistic Menger spaces. Nonlinear Anal. 71, 2734-2738 (2009)

28. Mihet, D: Fixed point theorems in probabilistic metric spaces. Chaos Solitons Fractals 41(2), 1014-1019 (2009)

29. Mihet, D: A note on a paper of Hicks and Rhoades. Nonlinear Anal. 65, 1411-1413 (2006)

30. O'Regan, D, Saadati, R: Nonlinear contraction theorems in probabilistic spaces. Appl. Math. Comput. 195, 86-93 (2008)

31. Saadati, R, Sedghi, S, Shobe, F: Modified intuitionistic fuzzy metric spaces and some fixed point theorems. Chaos Solitons Fractals 38, 36-47 (2006)

32. Schweizer, B, Sklar, A: Probabilistic Metric Spaces. Elsevier, Amsterdam (1983).

33. Sedghi, S, Žikič-Došenovič, T, Shobe, F: Common fixed point theorems in Menger probabilistic quasimetric spaces. Fixed Point Theory Appl. 2009, Article ID 546273 (2009)

34. Hadzi, O: Fixed point theorems for multivalued mappings in probabilistic metric spaces. Fuzzy Sets Syst. 88, 219-226 (1997)

\section{Submit your manuscript to a SpringerOpen ${ }^{\circ}$ journal and benefit from:}

- Convenient online submission

Rigorous peer review

- Immediate publication on acceptance

- Open access: articles freely available online

- High visibility within the field

- Retaining the copyright to your article 